\title{
EVOLUTIONARY SEQUENCES FOR BINARY STARS IN THE MASS RANGE 9 TO $40 \mathrm{M} \odot$
}

\author{
C. de Loore $(1,2)$ and J.P. De Greve(1) \\ (1) Astrophysical Institute - Vrije Universiteit Brussel, Belgium \\ (2) Astrophysics Research Group, University of Antwerp, RUCA, Belgium
}

\begin{abstract}
A set of evolutionary sequences for the two components of massive close binaries in the mass range 9 to $40 \mathrm{M} \odot$ for mass ratios 0.9 and 0.6 has been calculated. The used code accounts for mass transfer according to case $\mathrm{B}$, i.e. mass exchange after core hydrogen exhaustion, during semi-detached and contact phases. All models were computed for a single initial chemical composition $X=0.7, Z=0.02$. Stellar wind mass losses were taken into account, as well as convective core overshooting $(\alpha=0.25)$. The Roche lobe overflow was calculated according to a simplified hydrodynamical treatment. For the mixing of infalling material with the originally present layers the process of thermohaline mixing was adopted. It was assumed that $50 \%$ of the matter lost by the primary is accreted by the secondary.
\end{abstract}

\section{Introduction}

The existing sets of evolutionary sequences for close binary systems available in the literature cover only restricted mass and mass ratio ranges. No extensive homogeneous series of computations exists. Moreover, most published sequences only treat the detailed structure of the primary component. To remediate to the lack of a consistent set of models, and to allow interpretation of observations, we calculated a set of evolutionary sequences for the two components of massive close binary systems.

In order to allow comparison with single star models, we chose our parameters so that they match those of recently published single star models (Maeder, 1991)

So we computed a network of detailed models, simultaneously for both components, for masses between 9 and $40 \mathrm{M}_{\odot}$, for mass ratios $\mathrm{q}\left(=\mathrm{M}_{2} / \mathrm{M}_{1}\right), 0.6$ and 0.9 (see Table 1). The models were computed with our code (Prantzos et al. 1986), used in previous work, adapted to new information on input physics, structure parameters and physical processes.

\section{The Computer Code}

For the two crucial effects, the extent of the convective core and the stellar wind mass losses, the underlying basic physical principles are not very well known, and parameters governing the core extension and mass loss rates are derived from comparison with observations. But also this is still a matter of debate and arguments can be presented for the use of small or large parameters to model the overshooting of the core. For these 
calculations, the leading argument to adopt a moderate overshooting of $\alpha=0.25$, was to allow comparison with the models for single stars published by Maeder, 1991.

Table 1

\begin{tabular}{|rrr|}
\hline $\mathrm{M}_{1}$ & $\mathrm{M}_{2}$ & $\mathrm{P}_{\text {in }}$ (days) \\
\hline 9 & 8.1 & 3.95 \\
9 & 5.4 & 3.75 \\
12 & 10.8 & 4.90 \\
12 & 5.4 & 4.69 \\
15 & 13.5 & 5.78 \\
15 & 9.0 & 5.55 \\
20 & 18.0 & 7.23 \\
20 & 12.0 & 6.91 \\
25 & 22.5 & 8.94 \\
25 & 15.0 & 8.57 \\
30 & 27.0 & 14.00 \\
30 & 18.0 & 30.00 \\
40 & 36.0 & 30.00 \\
40 & 24.0 & 25.00 \\
\hline
\end{tabular}

Mass loss is included using the semi-empirical mass loss formalism of de Jager et al.(1991). Evolved stars with mass larger than $5 \mathrm{M} \odot$, with hydrogen exhausted in the core, and hydrogen abundance of the outer layers below 0.4 , are considered as Wolf-Rayet stars. Instead of using a constant mass loss rate as was done before, we adopted a Wolf-Rayet mass loss rate dependent on the mass (Langer, 1989)

$$
\dot{\mathrm{M}}=\mathrm{k}\left(\frac{\mathrm{M}_{\mathrm{WR}}}{\mathrm{M}_{\mathrm{o}}}\right)^{2.5} \text { with } \mathrm{k}=10^{-7}
$$

Opacities were taken from the Los Alamos Opacity Library (Huebner et al. 1977).For the equation of state we took into account gas-, degenerate- and radiative pressure. The detailed structure of each component with changing mass was computed as well as the variation of the orbital elements due to the mass changes (stellar wind, mass loss and accretion effects). Hydrodynamic effects and deviations from spherical symmetry, -rotational and tidal effects-, were neglected and the binary orbit was always assumed to be circular. The rotation of the components is supposed to remain synchronized with the orbital motion.

Mass leaving the system, as stellar wind or during mass exchange phases, will carry away some angular momentum. The behaviour of gas streams around and between the stellar components is still poorly known, and essentially of qualitative nature. To describe the mass and angular momentum transfer two parameters are adopted, one to describe the fraction of the matter lost by the primary and accreted by the secondary, and another to describe the angular momentum transfer. Mass loss and accretion rates are related as 


$$
\mathrm{dM}_{\mathrm{r}} / \mathrm{dt}=\beta \mathrm{d} \mathrm{M}_{\mathrm{d}} / \mathrm{dt}
$$

with $M_{r}$ and $M_{d}$ the masses of the receiver and the donor. We adopted for $\beta$ the value 0.5 , following the argumentation of Meurs and van den Heuvel (1989).

Stellar wind mass loss may be assumed to occur according to Jeans' mode (Huang, 1963). The change of period and separation is then calcalted as

$$
\frac{P}{P_{i}}=\left(\frac{M_{1 i}+M_{2 i}}{M_{1}+M_{2}}\right)^{2}
$$

with $\mathrm{M}$ and $\mathrm{P}$ the component mass, the total mass and the orbital period. During phases of mass transfer the change of the orbital angular momentum $\Delta \mathrm{J}$ can be calculated as

$$
\frac{\Delta \mathrm{J}}{\mathrm{J}}=1-\left(1-\frac{\Delta \mathrm{M}}{\mathrm{M}}\right)^{\gamma}
$$

with $\gamma$ a constant to be fixed. We used here $\gamma=2.1$ (De Greve et al. 1985)

The Roche lobe overflow is described by a simplified hydrodynamical treatment of the kind originally developed by Jedrzejec (see Paczynski and Sienkiewicz, 1972).
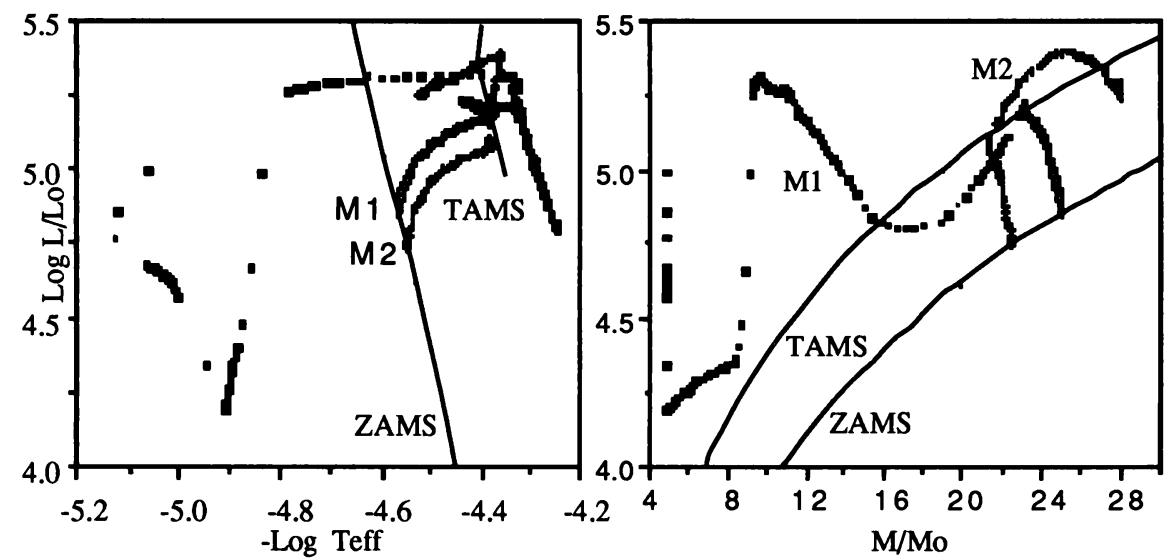

Fig.1. Left : Evolutionary tracks in the HRD of the two components of the system of $25 \mathrm{M} \odot+22.5 \mathrm{M} \odot$, with initial period of 8.94 days. Right : The mass-luminosity relation for both components of the $25 \mathrm{M} \odot$ $+22.5 \mathrm{M} \odot$ system.

\section{Results}

The results, available in the form of tables, show the stellar parameters for the stages : Zero Age Main Sequence, ZAMS, red point RP, i.e. the outermost redward position of the primary during core $\mathrm{H}$-burning, the point where the central $\mathrm{H}$ - abundance of the primary vanishes, Xc1, start of the mass transfer, Roche Lobe Overflow RLOFi, point where the atmospheric $\mathrm{H}$-abundance of the primary drops below its initial value, $\mathrm{Xat}<0.7$, point where core He-burning of the primary starts, i.e. CHeBIi, point where the mass transfer 
stops, RLOFf, stage of core He-exhaustion of the primary. Table 2 shows the results for a system of $25 \mathrm{M} \odot+22.5 \mathrm{M} \odot$ with initial period 8.94 days, and in Fig. 1 the evolutionary tracks in the HR diagram, and the M-L relation for both components. The full set will be published elsewhere (De Loore and De Greve, 1991).

Table 2

EVOLUTION $25 \mathrm{M} \odot+22.5 \mathrm{M} \odot-$ Period $=8.94 \mathrm{~d}$

\begin{tabular}{|c|c|c|c|c|c|c|c|c|c|c|}
\hline \multirow[t]{2}{*}{ Phase } & Age (106yr) & ) M1 & Mass & $\log \mathrm{T}_{\mathrm{e} 1}$ & \multirow{2}{*}{$\begin{array}{l}\log \mathrm{L} 1 \\
\log \mathrm{L} 2\end{array}$} & \multirow{2}{*}{$\begin{array}{l}\mathrm{X} 11 \\
\mathrm{X} 22\end{array}$} & \multirow{2}{*}{$\begin{array}{l}\text { Yc1 } \\
\text { Yc2 }\end{array}$} & \multirow{2}{*}{$\begin{array}{l}\text { Xat } 1 \\
\text { Xat } 2\end{array}$} & \multirow{2}{*}{$\begin{array}{l}\text { Rad1 } \\
\text { Rad2 }\end{array}$} & \multirow{2}{*}{$\begin{array}{l}\text { Mcc1 } \\
\text { Mcc2 }\end{array}$} \\
\hline & & M2 & loss & $\log \mathrm{T}_{\mathrm{e} 2}$ & & & & & & \\
\hline \multirow[t]{2}{*}{ ZAMS } & 02 & 25.0 & $\mathrm{E}+0$ & 5 & 4.853 & .70 & .280 & .700 & .58 & 13.91 \\
\hline & 940 & 22.5 & $0.00 \mathrm{E}+0$ & 4.549 & 4.739 & .70 & .280 & .700 & 6.22 & 12.18 \\
\hline \multirow[t]{2}{*}{ RP1 } & 8.181 & 23.3 & $-0.55 E-6$ & $5 \quad 4.377$ & 5.186 & .02 & .964 & .700 & 22.97 & 7.96 \\
\hline & 10 & 21.4 & -0.3 & $5 \quad 4.394$ & 5.066 & .08 & .899 & .700 & & .31 \\
\hline \multirow[t]{2}{*}{$\mathrm{X} \subset 1=0$} & & 23.2 & -0 & $5 \quad 4.409$ & 04 & .00 & .979 & .700 & 29 & .69 \\
\hline & & 21.4 & -0 . & $5 \quad 4.383$ & 5 & .06 & .922 & .700 & 63 & 7.16 \\
\hline \multirow[t]{2}{*}{ RLOFi } & & 23.2 & -0 . & 54.3 & 2 & .00 & .980 & .700 & 80 & .00 \\
\hline & & 21.4 & -0 . & 54. & 16 & .05 & .927 & .700 & 86 & 7.13 \\
\hline \multirow[t]{2}{*}{ Xat $<0.7$} & 8 & 16.3 & -0 . & 4. & 1 & .00 & .980 & .690 & 27 & .00 \\
\hline & 10. & 24.8 & 0 & 4. & & .05 & .926 & .700 & 31 & 7.57 \\
\hline \multirow[t]{2}{*}{ CHeB1i } & & 11.0 & -0 . & 8 & 3 & .00 & .979 & .368 & 30 & 2.39 \\
\hline & 15. & 27.4 & 0 . & 4.458 & 5.2 & .06 & .918 & .677 & 18 & 8.22 \\
\hline \multirow[t]{2}{*}{ RLOFf } & 7 & 9.7 & -0 . & 4.401 & 5.3 & .00 & .971 & .339 & 23 & 5.16 \\
\hline & 17. & 28.0 & -0.4 & 4.505 & 5.260 & .17 & .810 & .665 & 14.06 & 9.63 \\
\hline \multirow[t]{2}{*}{$Y \subset 1=0$} & & 4.9 & $8+0$ & 5.123 & 4.765 & .00 & .000 & .000 & .46 & .00 \\
\hline & 24.068 & 27.6 & $-0.58 E-6$ & 4.483 & 5.283 & .13 & .852 & .665 & 15.75 & 10.55 \\
\hline
\end{tabular}

\section{References}

De Greve, J.P., de Landtsheer, A.C., Packet, W., 1985, Astron. Astrophys.142, 367 Huang, S.-S., 1963, Astrophys.J. 304, 695

Huebner, W.F., Merts, A.L., Magee, N.H., Argo, M.F., 1977, Los Alamos Sci. Lab. Rept. LA-66760-M

Langer, N, 1989, Astron. Astrophys.220, 135

de Loore. C., De Greve, J.P., 1992, Astron. Astrophys. submitted

Maeder, A., 1991, preprint

Meurs, E.J.A., van den Heuvel, E.P.J., Astron. Astrophys.226, 88

Prantzos, N., Doom, C., Arnould, M , de Loore, C., 1986, Astrophys.J. 304, 695

Paczynski, B., Sienkiewicz, 1972, Acta Atron. 22, 73 